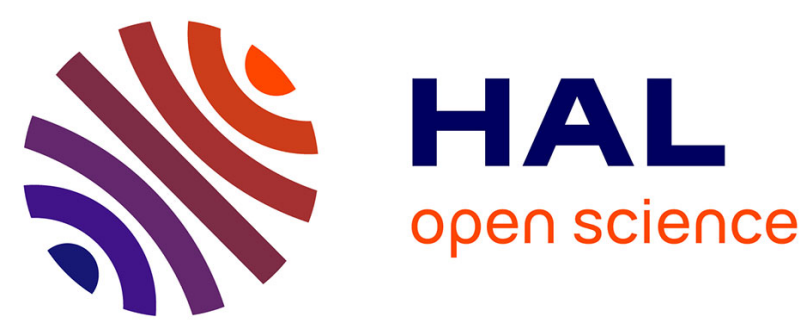

\title{
A low cost, handheld E-nose for renal diseases early diagnosis
}

Paul Le Maout, Pierre S Laquintinie, Cyril Lahuec, Fabrice Seguin, J-L Wojkiewicz, N. Redon, Laurent Dupont

\section{- To cite this version:}

Paul Le Maout, Pierre S Laquintinie, Cyril Lahuec, Fabrice Seguin, J-L Wojkiewicz, et al.. A low cost, handheld E-nose for renal diseases early diagnosis. 2018 40th Annual International Conference of the IEEE Engineering in Medicine and Biology Society (EMBC), Jul 2018, Honolulu, United States. 10.1109/EMBC.2018.8512847 . hal-01915321

\section{HAL Id: hal-01915321 \\ https://hal.science/hal-01915321}

Submitted on 7 Nov 2018

HAL is a multi-disciplinary open access archive for the deposit and dissemination of scientific research documents, whether they are published or not. The documents may come from teaching and research institutions in France or abroad, or from public or private research centers.
L'archive ouverte pluridisciplinaire HAL, est destinée au dépôt et à la diffusion de documents scientifiques de niveau recherche, publiés ou non, émanant des établissements d'enseignement et de recherche français ou étrangers, des laboratoires publics ou privés. 


\title{
A low cost, handheld E-nose for renal diseases early diagnosis
}

\author{
P. Le Maout*, P.S Laquintinie*, C. Lahuec*, F. Seguin*, J-L. Wojkiewicz ${ }^{\dagger}$, N. Redon ${ }^{\dagger}$, L. Dupont \\ ${ }^{*}$ Electronics Dept., ${ }^{\ddagger}$ Optics Dept. IMT Atlantique \\ CNRS Lab-STICC UMR 3192, Technople Brest Iroise - CS 83818, 29238 BREST CEDEX 3, France \\ Email: cyril.lahuec@imt-atlantique.fr \\ $\dagger$ †SAGE Dept., IMT Lille Douai, CS10838 59508 DOUAI, France \\ Email: jean-luc.wojkiewicz@imt-lille-douai.fr
}

\begin{abstract}
In the last decade, the use of electronic olfaction systems for the early diagnosis of several pathologies by breath analysis has been investigated. In this study, an electronic nose including seven polyaniline sensors has been developed. An impedance measurement circuit and a micro-computer to process the sensor responses were studied to give a prediagnosis conclusion. The measurement accuracy is $97 \%$ when it is exposed to a simulated human breath and different concentration of ammonia, from $500 \mathrm{ppb}$ to $2.8 \mathrm{ppm}$. The described prototype weights about $300 \mathrm{~g}$ and can be used for 14 hours with a smartphone battery.
\end{abstract}

\section{INTRODUCTION}

With $10 \%$ of the global population affected by chronic kidney disease [1], the development of a non-invasive, easy to use and cost-effective solution to diagnose kidney pathologies is of utmost importance. In order to check patients for kidney diseases two tests are currently in use: a blood and urine tests. The first one assesses the glomerular filtration rate while the second one the abnormal presence of proteins. Both of these procedures are simple and cost-effective but require specific operator and laboratory. Moreover, blood tests are invasive. Studies have shown that patients with renal diseases have higher amount of ammonia in breath than healthy ones [2]. Analysing breath composition could lead to simple diagnosis tools to diagnose kidney diseases. As human breath is a complex mixture of gases and volatile compounds. It has been shown that combining cross sensitive sensors with classification algorithms is a very effective method to associate a pattern response of sensor to a disease [3]. Such systems are referred to as electronic noses (E-nose). They are composed of:

1) an array of sensors chosen for their cross-sensitivity to chemical compounds of interest,

2) a signal processing stage to extract relevant features from the sensors signals,

3) a classification algorithm to associate the sensor array responses to an healthy or ill breath.

Different electronic noses already exist. However, they are usually not fully handheld systems as they require a personal computer type system to exploit the measurements [4]. Another drawback is their cost, about few thousands of dollars.

This work was supported by Institut Mines Telecom Fundation in the "Future et Ruptures" program. And by the Direction Generale de l'Armement (DGA thesis number 2016337)
See for instance the Cyranose $320^{\circledR}$ [5]. This paper presents a low cost efficient handheld E-nose for early diagnosis of renal diseases. The simulated human breath which is used to test the system is described in section II. Section III explains the choices made to design the proposed E-nose. Section IV and V describe the experimentation and its results respectively. Section VI concludes the paper.

\section{HUMAN BREATH}

Human breath contains hundreds of compounds. Concentration of compounds ranges from few ppt up to thousands of $\mathrm{ppb}$ [6]. The variability of the composition between individuals is important, and depend on many factors as age, gender or health condition. A standard composition of a simulated human breath has been made, based on mean concentration given in [6][7]. Its composition is : $800 \mathrm{ppb}$ of ammonia $( \pm 10 \%), 500 \mathrm{ppb}$ of acetone $( \pm 0.5 \%), 500 \mathrm{ppb}$ of methanol $( \pm 1 \%), 200 \mathrm{ppb}$ of ethanol $( \pm 0.5 \%), 100 \mathrm{ppb}$ of isoprene $( \pm 5 \%)$ and $20 \mathrm{ppb}$ of acetaldehyde $( \pm 5 \%)$. Relative humidity during injection increases to $70 \%$. Carbon dioxide is not added because it does not react with the formulated sensors.

\section{Proposed Electronic NOSE SyStem}

The proposed electronic nose is based on a single-board computer, a Raspberry Pi. Its low cost, size, computational power and various of available interfaces (I2C, GPIO, SPI) made it a versatile and powerful control unit for the electronic nose. Both the measurement Front End and Raspberry Pi have the size of a credit card as shown in Figure 2. The developped sensor array is $2.5 \times 0.5 \times 0.1 \mathrm{~cm}^{3}$. Packaging the complete system costs about $50 \$$, and weights $300 \mathrm{~g}$. Therefore, a handheld E-nose is then possible.

\section{A. Custom Ammonia Sensors}

A good candidate to detect the target compound, ammonia, in the ppb range, is the polyaniline (PANI). It is an intrinsically conductive polymer. Moreover, PANI is a low cost material. When in presence of ammonia, the equivalent electrical impedance varies in time. The electrical model of a polyaniline sensor is a resistance and a capacitive element in parallel, Figure 1a [8]. By changing the structure of the polymer matrix, and surface/volume ratio between formulations, different impedance variations, i.e. differentiated 


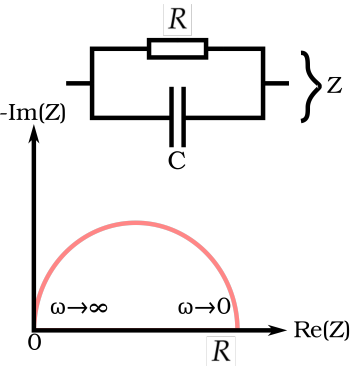

(a)

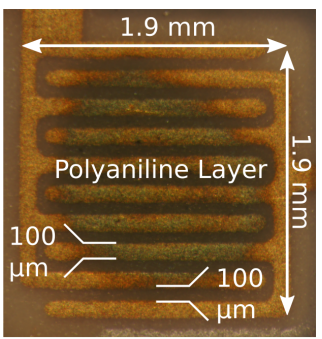

(b)
Fig. 1. (a) Equivalent electrical model of the sensor and its Nyquist diagram are shown. (b) Polyaniline sensor and interdigitated electrode dimensions.

responses, are observed. The sensors were manufactured by drop coating a polyaniline solution on gold interdigitated electrodes deposited on an ceramic substrate, Figure 1b. The worst case sensors sensitivity is in the order of $5 \%$ impedance variation per ppm when equilibrium is reached. Seven PANI sensors with different formulation are used [9][10], which is enough for correct olfactive pattern recognition [3].

\section{B. Front End: Impedance measurement}

Since the impedance of the sensors is complex, it is chosen to use the AD5933 impedance measurement integrated circuit from Analog Device. The circuit has built-in calibrations for impedance measurements and temperature. Moreover, the AD5933 has an I2C port for communication with Raspberry $\mathrm{Pi}$. The impedance to measure is excited with a sinusoidal voltage signal at $1 \mathrm{kHz}$ and $30 \mathrm{kHz}$. The sensor response signal is then sampled at a rate of $2 \mathrm{~Hz}$ (controlled by the Raspberry) for computing the real and imaginary parts of the impedance. These values are then communicated to the Raspeberry via the I2C port. The measurement accuracy of the AD5933 is $0.5 \%$. It yields an error of concentration measurement of $100 \mathrm{ppb}$, in line with the sensors sensitivity. To measure the impedance of the seven sensors, a 8:1 analog multiplexer (ADG708) is inserted between the AD5933 and the sensor array. The $8^{\text {th }}$ multiplexer input is used for impedance calibration purposes.

\section{Feature extraction}

Sensors responses processing is done on the Raspberry Pi. The first step consists in filtering the curves with a moving average operation to reduce the noise and remove any incoherent measures. The resistance is then calculated from the real and imaginary values provided by the measurement front-end circuit:

$$
R=\frac{\operatorname{Re}^{2}(Z)+\operatorname{Im}^{2}(Z)}{\operatorname{Re}(Z)}
$$

where $\operatorname{Re}(Z)$ and $\operatorname{Im}(Z)$ are the real and the imaginary parts of the impedance $Z$, respectively. Computed $R$ values are stored in memory before features are extracted from the memorized points. Typically, only the maximum variation is extracted from the curve, Figure 3. However, it has been shown that transient features such as integrals

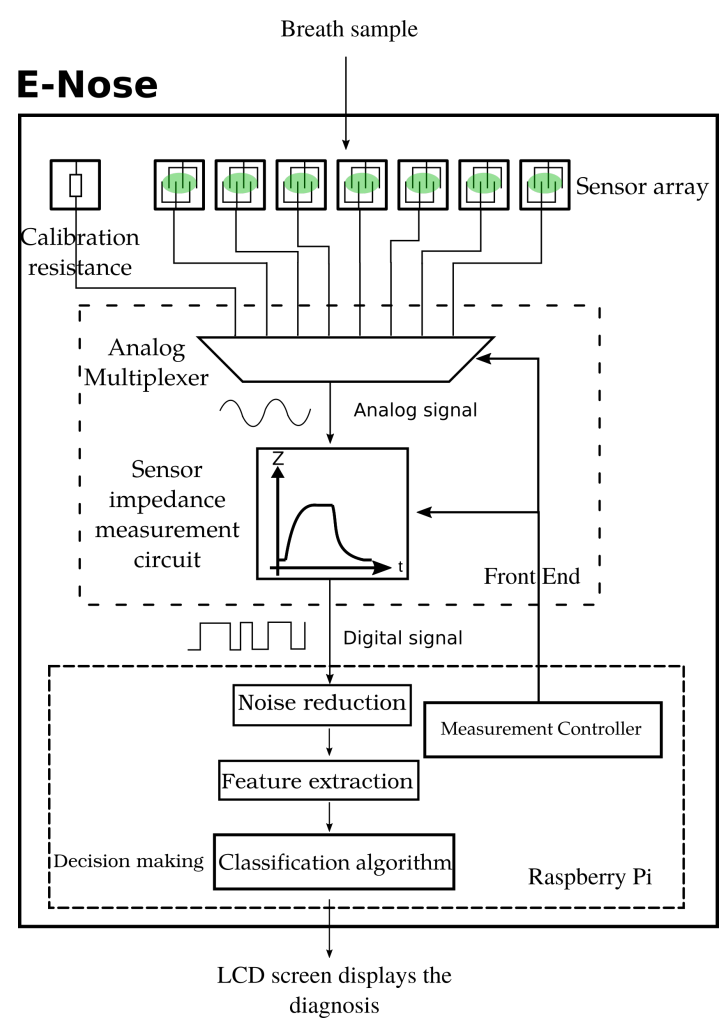

(a)

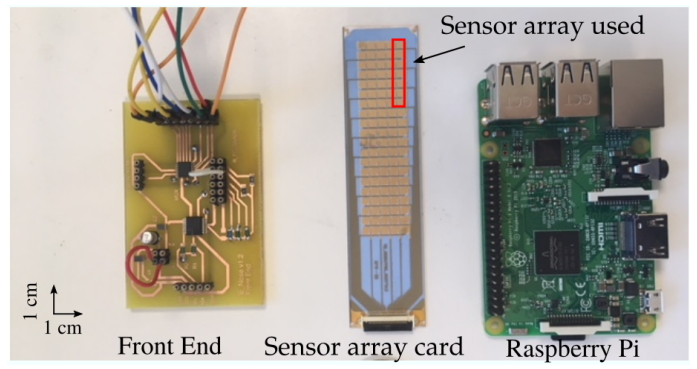

(b)

Fig. 2. (a) Architecture of the electronic nose system and (b) the picture of different parts of the electronic nose. The sensor array card is composed of 100 sensors even if only the seven outlined ones are used.

and/or derivative could also help increase the classification performance [11]. The set of features extrated is thus:

- the maximum derivative of the signal: $\delta_{R}$,

- the integral value: $\int R$,

- the maximum variation: $\Delta_{R}$.

For each sensor, features are extracted during both the adsorption and desorption phases, Figure 3, yielding a total of 42 features (six extracted features for the seven sensors).

\section{Classification stage}

Typically, the classification is performed in two steps. First, the set of extracted features is transformed into a reduced set of dimensions by means of a specific algorithm. Second, the actual classification algorithm works on the reduced set of dimensions. The classification process required 


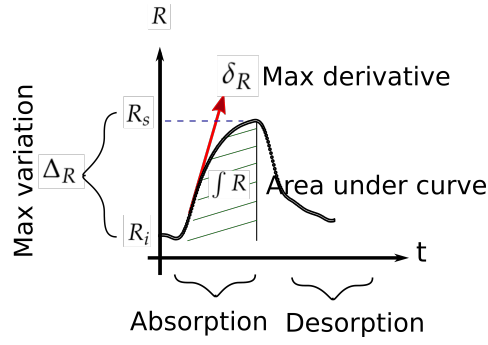

Fig. 3. Typical gas sensor response with the different features extracted.

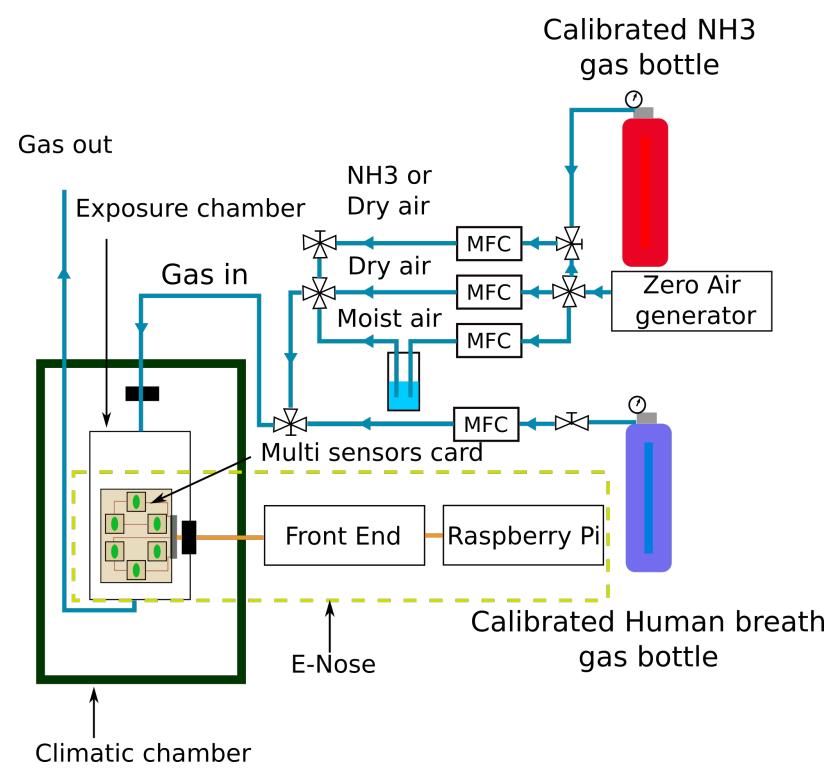

Fig. 4. Illustration of the E-nose laboratory test bench.

training of both algorithms to discriminate different classes. Several algorithms exist for dimension reduction, Principal Component Analysis (PCA), Linear Discriminant Analysis (LDA) as well as classification algorithms, LDA, support Vector Machine (SVM), Artificial Neural Networks (ANN) and Random Forest (RF) algorithm, to name the most used. Several combinations are possible. Since predicting which one yields the best results is impossible, testing is necessary [12]. Validating a classifier requires to randomly separate the data into training and testing databases. Typically, the training database represents $60 \%$ of the data randomly chosen. This selection process is eased on the Rasberry Pi card thanks to the Python Scikit-Learn librairie having many algorithms implemented.

\section{EXPERIMENTAL SET-UP}

Moisted air, simulated human breath and ammonia are mixed and injected for five minutes into the exposure chamber in which the sensor array is. The amount of ammonia added in the mixture varies from $500 \mathrm{ppb}$ to $2 \mathrm{ppm}$. Three classes are defined: Healthy, Uncertain, and Unhealthy. They correspond to three concentration ranges of ammonia : 500 ppb - $1.1 \mathrm{ppm}, 1.1 \mathrm{ppm}-1.7 \mathrm{ppm}, 1.7 \mathrm{ppm}-2.8 \mathrm{ppm}$, respectively. The exposure chamber is in a climatic room, with a controled temperature of $27^{\circ} \mathrm{C}$, Figure 4 . The flow rate is at $8 \mathrm{~L} \cdot \mathrm{min}^{-1}$. After an exposition, a purified and dry air is injected during five minutes. A total of 100 cycles are then realised with non specific order in the concentration of ammonia added in the mixture. An ammonia analyser and a humidity sensor is used to control the real concentration of ammonia and the relative humidity inside the exposure chamber.

\section{RESUlts}

\section{A. Sensor responses}

Figure 5 shows typical responses of 2 different sensors. Exposed to a mixture of simulated human breath and $1.5 \mathrm{ppm}$ of ammonia, sensors have similar responses but different $\Delta_{R}$ , $\delta_{R}$ and $\int_{R}$ (table I). For a same concentration of ammonia, sensor responses vary. For exemple, the resistance variation for sensor 2 varies from $6.1 \%$ to $9 \%$ for $2 \mathrm{ppm}$ of ammonia. Thus, a predicted concentration error of $600 \mathrm{ppb}$ is possible. This is enough to mistake a healthy sample as a unhealthy one.

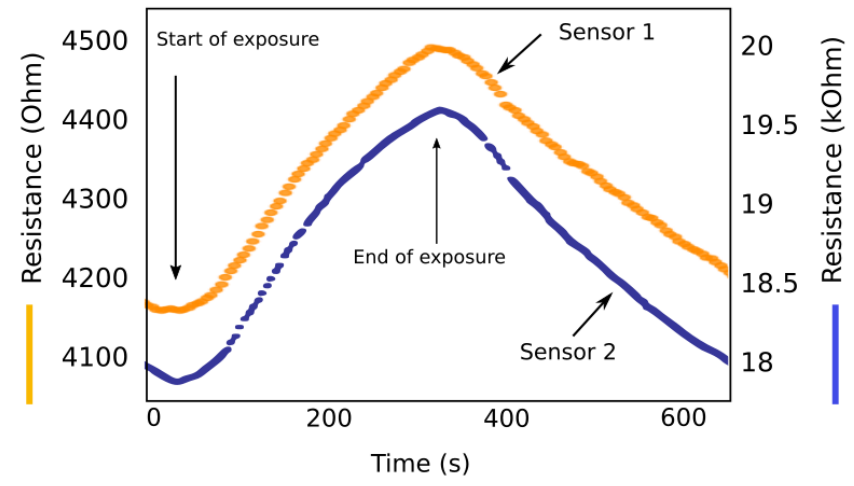

Fig. 5. Resistance variation of sensors 1 and 2 to $1.5 \mathrm{ppm}$ of ammonia and human breath composition.

TABLE I

RESPONSE FEATURES OF SENSORS 1 AND 2 TO 1.5 PPM OF AMMONIA.

\begin{tabular}{|c||c|c|c||c|c|c|}
\hline \multicolumn{1}{|c||}{} & \multicolumn{3}{c||}{ Absorption } & \multicolumn{3}{c|}{ Desorption } \\
\hline & $\Delta_{R}(\%)$ & $\delta_{R}$ & $\int_{R}$ & $\Delta_{R}(\%)$ & $\delta_{R}$ & $\int_{R}\left(\times 10^{3}\right)$ \\
\hline Sensor 1 & 8.4 & 99 & 372 & -5.5 & -79 & 358 \\
\hline Sensor 2 & 7.3 & 12 & 86 & -6.7 & -9 & 83 \\
\hline
\end{tabular}

\section{B. Classication results}

After testing various combinations of dimension reduction and classification algorithms, the best combination is LDA and SVM, respectively. LDA reduces the number of dimensions from 42 down to two by linearly combining the original features. Figure 6 shows the projection of the database with LDA algorithm and is already efficient to discriminate the different classes with a classification accuracy of 93\%. Then SVM algorithm is applied to the 2-D dataset. SVM principle is to calculate an hyperplan that segregate at best the different classes. The SVM algorithm classifies the samples with an accuracy of prediction of $97 \%$. These results are detailed 


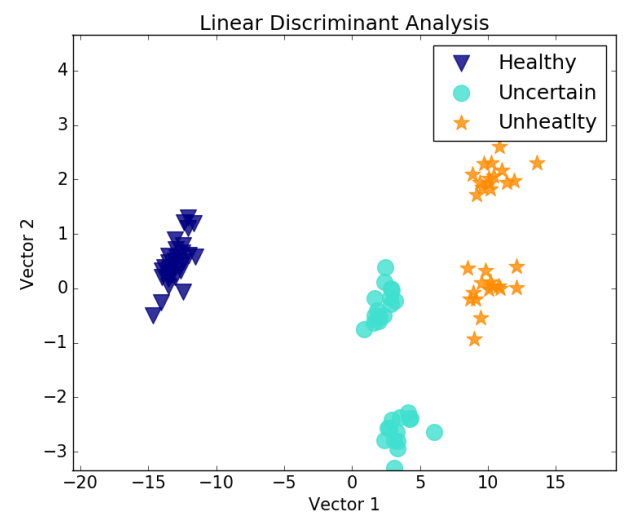

Fig. 6. Application of the linear discriminant algorithm.

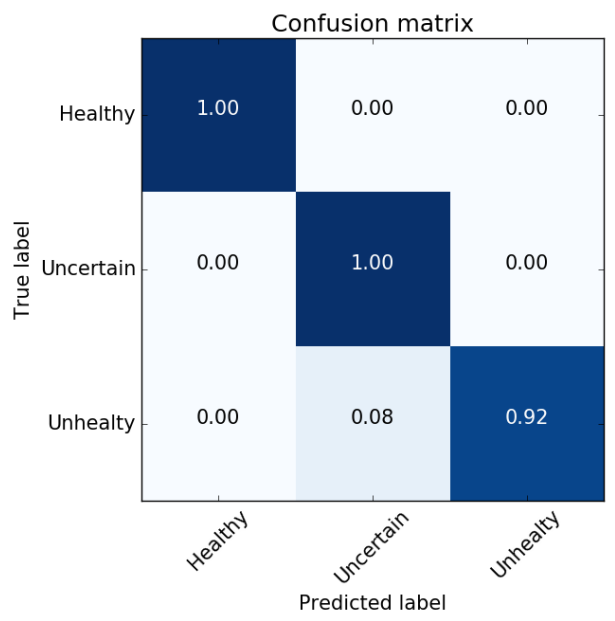

Fig. 7. Normalized confusion matrix of the SVM algorithm. Healthy and Uncertain samples are always well predicted. Unhealthy ones are correctly classified at $92 \%$.

on the confusion matrix in Figure 7: row gives the class of a tested sample, and column the class predicted by the classifier. Accuracy is calculated by dividing the sum of correctly classified samples by the total sample tested. In the present case, a healthy or uncertain sample is always correctly classified. The confusion matrix points out that the predicted errors are only with unhealthy samples: $92 \%$ are accurately classified but $8 \%$ are predicted as uncertain. This is in line with the LDA results in Figure 6, where unhealthy and uncertain groups are close from each other. Errors between healthy/unhealthy would be more problematic in a diagnosis help system. Crossing responses from several sensors based on PANI with a classification stage is thus appropriate to reduce the error.

When used without dimensional reduction with the 42-D vectors, the SVM accuracy drops to $94 \%$. The use of LDA for dimensionnal reduction is therefore necessary. Moreover it allows an easier hardware implantation of the classification stage. Further works will focus on confronting the proposed system with a more diverse set of simulated breath samples and real human breath.

\section{System power requirements}

The power consumption of the system is $1.35 \mathrm{~W}$ when idle, $1.5 \mathrm{~W}$ during measurement and $2.1 \mathrm{~W}$ during classification stage. The measurement front-end power consumption is only $0.1 \mathrm{~W}$ so the next step is to optimize the controller and classification parts with a more dedicated circuit.

For instance, this electronic nose prototype could be powered for 14 hours with a $5000 \mathrm{mAh}$ battery.

\section{CONCLUSIONS}

The electronic nose system presented in this paper allows detecting different levels of ammonia concentration in a simulated human breath with an accuracy of $97 \%$.

Moreover, it is a low cost, portable and easy to use diagnosis system for a general practitioner. Such a system could allow a broader access to point of care testing for populations living in areas lacking of medical care services. Further work will heavily focus on further reducing the size and power consumption of the system by proposing a hardware implantation of all its components.

\section{REFERENCES}

[1] G. facts about kidney disease, "www.kindney.org," 2018. [Online]. Available: https://www.kidney.org/kidneydisease/global-facts-aboutkidney-disease

[2] S. Davies, P. Spanel, and D. Smith, "Quantitative analysis of ammonia on the breath of patients in end-stage renal failure," Kidney International, vol. 52, no. 1, pp. 223-228, July 1997.

[3] S. M. Scott, D. James, and Z. Ali, "Data analysis for electronic nose systems," Microchimica Acta, vol. 156, no. 3-4, pp. 183-207, Dec. 2006.

[4] S. W. Chiu, J. H. Wang, G. T. Lin, C. L. Chang, H. Chen, and K. T. Tang, "Towards a fully integrated electronic nose SoC," in 2012 IEEE 55th International Midwest Symposium on Circuits and Systems (MWSCAS), Aug. 2012, pp. 166-169.

[5] Cyranose320, "Sensigent company." [Online]. Available: http://www.sensigent.com/products/cyranose.html

[6] M. Phillips, J. Herrera, S. Krishnan, M. Zain, J. Greenberg, and R. N. Cataneo, "Variation in volatile organic compounds in the breath of normal humans," Journal of Chromatography B: Biomedical Sciences and Applications, vol. 729, no. 12, pp. 75-88, June 1999. [Online]. Available: http://www.sciencedirect.com/science/article/pii/S0378434799001279

[7] C. Turner, P. panl, and D. Smith, "A longitudinal study of methanol in the exhaled breath of 30 healthy volunteers using selected ion flow tube mass spectrometry, SIFT-MS," Physiological Measurement, vol. 27, no. 7, p. 637, 2006. [Online]. Available: http://stacks.iop.org/0967-3334/27/i=7/a=007

[8] C. Vaghela, M. Kulkarni, S. Haram, M. Karve, and R. Aiyer, "Biopolymer-Polyaniline Composite for a Wide Range Ammonia Gas Sensor," IEEE Sensors Journal, vol. 16, no. 11, pp. 4318-4325, June 2016.

[9] T. Merian, N. Redon, Z. Zujovic, D. Stanisavljev, J. L. Wojkiewicz, and M. Gizdavic-Nikolaidis, "Ultra sensitive ammonia sensors based on microwave synthesized nanofibrillar polyanilines," Sensors and Actuators B: Chemical, vol. 203, pp. 626-634, Nov. 2014.

[10] S. Mikhaylov, N. A. Ogurtsov, N. Redon, P. Coddeville, J.-L. Wojkiewicz, and A. A. Pud, "The PANI-DBSA content and dispersing solvent as influencing parameters in sensing performances of $\mathrm{TiO}_{2}$ /PANI-DBSA hybrid nanocomposites to ammonia," RSC Adv., vol. 6 , no. 86, pp. 82 625-82 634, 2016.

[11] K. Yan and D. Zhang, "Feature selection and analysis on correlated gas sensor data with recursive feature elimination," Sensors and Actuators B: Chemical, vol. 212, pp. 353-363, June 2015.

[12] J. H. Leopold, L. D. J. Bos, P. J. Sterk, M. J. Schultz, N. Fens, I. Horvath, A. Bikov, P. Montuschi, C. Di Natale, D. H. Yates, and A. Abu-Hanna, "Comparison of classification methods in breath analysis by electronic nose," Journal of Breath Research, vol. 9, no. 4, p. 046002 , Dec. 2015. 